\title{
Underground Cryogenic Detectors
}

\author{
Ettore Fiorini
}

\author{
Dipartimento di Fisica dell' Università di Milano \\ Sezione di Milano dell'Istituto Nazionale di Fisica Nucleare
}

The joint effort of cryogenics, particle physics, and astrophysics to exploit thermal detectors should lead to exciting and perhaps unexpected results.

Fundamental physics, having successfully unified the electromagnetic and weak interactions, is now attempting to unify the strong force and hopefully even the gravitational force. The so-called astro-particle physics connection is destined to play an important rôle. Searches with high-energy particle accelerators can be complemented in a wonderful way by studying, for example, the origin of the Universe and its remnants, the physical processes accompanying the origin, and the life and the death of a star. The astrophysics approach has led to the construction of large laboratories placed deep underground to investigate the interactions of the penetrating component of cosmic rays as well as the rare processes arising from the spontaneous decay of particles or nuclei. Noise from cosmic rays is suppressed because the rays are absorbed by the rock overburden.

The astrophysical approach has also stimulated the development and application of techniques which are either totally new or at least seldom or never used in particle physics. The thermal detection of nuclear radiation, perhaps the most promising of these techniques, played an important rôle in the development of nuclear physics. In 1903, Curie and Laborde published a paper entitled Le chaleur dégagée spontanément par les sels de radium (The heat released by radium salts) and in 1927 Ellis and Wooster found in the decay of ${ }^{210} \mathrm{Bi}$ missing thermal energy which was subsequently attributed by Pauli to the emission of neutrinos. The thermal detection of single particles was suggested independently in Europe [1] and the USA [2] for experiments in elementary particle physics some eight years ago. Since then, the development of these techniques has been impressive [3] and their application in experiments of the passive type [4], namely without accelerators, seems almost within reach.

\section{Techniques}

Some of the thermal techniques for detecting elementary particles are based on the effect that the thermal "action" of a particle may play on a metastable system. Con-

Ettore Fiorini is Professor of Advanced Physics and Director of the high-energy physics group in the Physics Department, University of Milan. He studied at the university and has worked at Duke University in the USA, and at the Paul Scherrer Institute and CERN in Switzerland. His group participates in Gran Sasso's GALLEX experiment but is mainly involved in developing cryogenic thermal detectors.
B

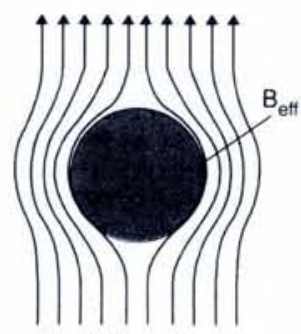

Superconducting

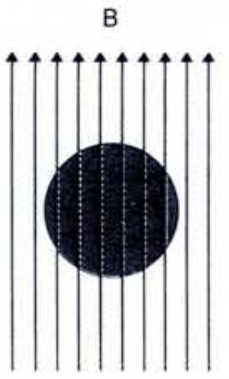

Normal conducting sider, for instance, a granule of a Type I superconductor kept at a fixed temperature in a magnetic field slightly below the critical field. The granule is in a metastable superconductor state and as such repels the magnetic field owing to the Meissner effect (Fig. 1a). Heat delivered by an incoming particle raises the temperature of the granule, bringing it suddenly to the normal state and the field is no longer repelled. The result is a magnetic pulse that can be detected.

Another technique, based on the Josephson effect, exploits a detector comprising a pair of superconductors separated by a thin, insulating layer. The superconductors induce breaking of the Cooper pairs to give a current pulse passing through the insulator which is amplified and measured (Fig. 1b).

More appropriate for "underground cryogenics" would appear to be another and in some ways simpler technique, which like the others, is generally called bolometric. Consider a very pure diamagnetic and dielectric crystal: its heat capacity $C_{v}$ (in joules per degree Kelvin) at low temperatures is given quantitatively by the expression:

$$
C_{\mathrm{v}}=1944\left(V / V_{\mathrm{m}}\right)\left(T / T_{\mathrm{D}}\right)^{3}
$$

where $V$ and $V_{m}$ are the crystal and molecular volumes, and $T$ and $T_{\mathrm{D}}$ are the operating and Debye temperatures. It is clear that for $T_{\mathrm{D}}$ sufficiently large and $T$ sufficiently small, the heat capacity can be so small that even the minute amount of energy delivered by an incident particle in the form of heat can produce, in the crystal, a sizable increase of temperature. This temperature pulse can be transformed into an electric pulse using a suitable thermistor in thermal contact with the crystal (Fig. 2). The thermistor, whose resistance we call $R_{\mathrm{b}}$, is biased by a battery and a load resistor with resistance $R_{L}$ possibly considerably larger than $R_{\mathrm{b}}$. The energy delivered by the particle to the crystal produces a negative voltage pulse across the thermistor which can then be amplified and measured.

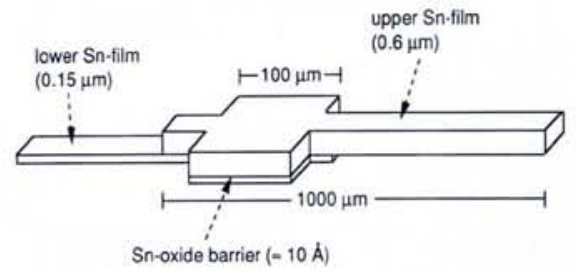

Fig. 1 - Techniques based on superconductivity for detecting fundamental particles: schematic illustrations of flux exclusion from a granule (a, left) and of a Josephson-type detector (b, right).

\section{Performance}

When comparing cryogenic detectors with standard detectors based semiconductors and on ionization or proportional counters, one should note that the standard devices collect only the fraction of energy delivered by the particle in form of ionization (about $30 \%$ of the total energy). The remainder of the energy goes mainly into phonons (heat), with perhaps a component into lattice dislocations in the case of nuclear recoils. Thermal detectors collect directly and in the form of heat at least $70 \%$ of the energy delivered, and up to $100 \%$ if the electron-ion and electron-hole pairs recombine within a sufficiently small time interval. Bolometers have in fact proved to be excellent detectors of nuclear recoils, which deliver a much lower fraction of energy as ionization than fast, charged particles, and consequently a much larger fraction in the form of heat. However, thermal detectors are "slow" since the rise time is related to the velocity of sound (the
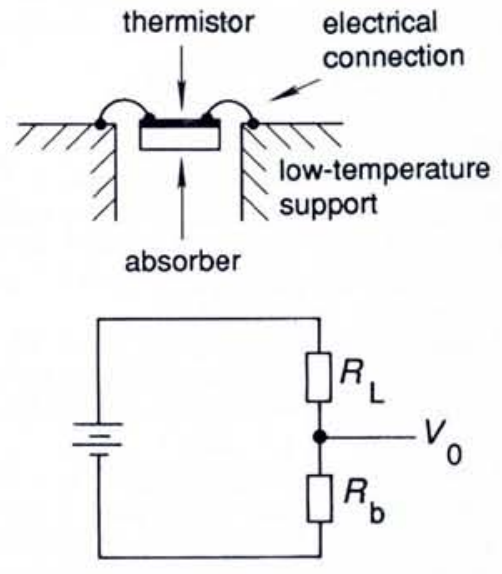

Fig. 2 - A schematic illustration of a bolometer and its electronic circuit which transforms a thermal pulse into a detectable electrical signal. 

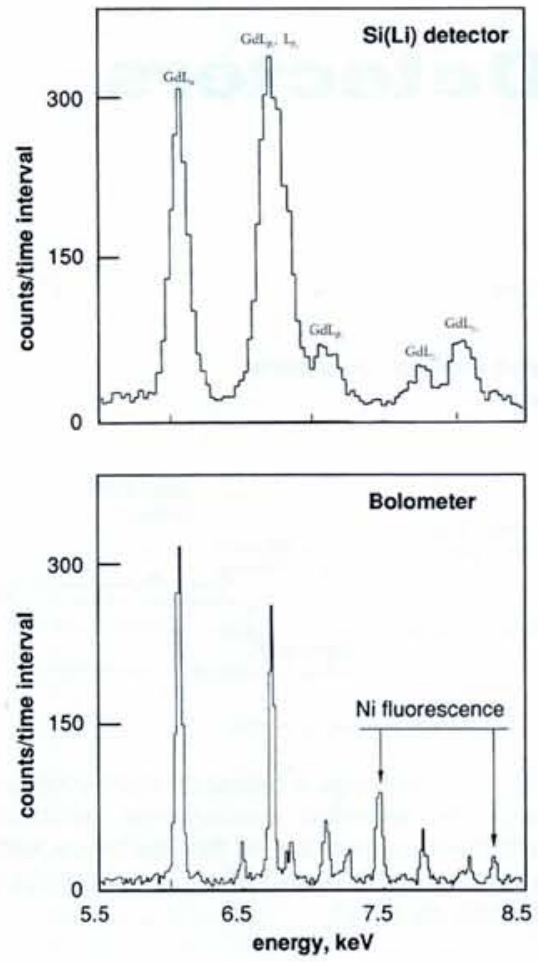

Fig. $3-A$ comparison between the $x$-ray spectroscopic performance of a Si $(\mathrm{Li})$ diode (upper) with that for the NASA-Wisconsin $158 \mathrm{~Tb}$ detector (lower). The bolometric device gave a resolution which is some 20 times better than for the semiconductor detector.

time needed for heat to distribute itself in the entire crystal), while the decay time is related to the thermal resistance between the bolometer and the heat sink. Thermal detectors are consequently excellent for searching for rare events in underground experiments, but usually unsuitable for working at the high counting rates and large backgrounds found at accelerators.

An important figure-of-merit for thermal detectors is the energy resolution. It can be optimized by adapting the characteristics of the preamplifier to the impedances of the bolometer and the load resistors, and by choosing the best value for the difference between the temperatures of the bolometer and the heat sink. The overall resolution of the detector (full width at the half maximum: FWHM) can be as small as:

$$
\Delta E=2.36 \xi \sqrt{ } k C_{v} T^{2}
$$

where $k$ is Boltzmann's constant and $\xi$ is a dimensionless parameter usually of the order of a few unities. The following examples demonstrate that the resolution should be, in principle, much better than for any other detector:

- A cubic crystal of silicon ( $T_{D}=645 \mathrm{~K}$ ) of $1 \mathrm{~mm}$ side kept at $20 \mathrm{mK}$ would have a heat capacity of $5 \times 10^{-15} \mathrm{~J} / \mathrm{K}$ and a FWHM resolution of $0.1 \mathrm{eV}$.

- A germanium thermal detector $\left(T_{D}=370\right.$ K) with $100 \mathrm{~mm}$ side (more than $5 \mathrm{~kg}$ in weight) operated at the same temperature would have an heat capacity around $2.5 \times$ $10^{-8} \mathrm{~J} / \mathrm{K}$ and a resolution of about $150 \mathrm{eV}$. Both the overall mass and the resolution would be much larger than for existing germanium diodes.
Unfortunately, we are still quite far from these limits owing to various important problems including:

- non-uniformity in collecting phonons, especially in large detectors;

- spatial non-uniformity of the recombination of electron-hole pairs trapped by various impurities;

- noise due to electromagnetic sources, especially microphonics [5];

- difficulties in keeping constant the temperature of the bolometer, and consequently its gain.

\section{Results}

Despite these difficulties the performance of some thermal detectors is already very promising. In the limit of very small detectors (where the heat capacity is obviously intrinsically very small), a NASA-University of Wisconsin collaboration recently achieved a resolution of $7.3 \mathrm{eV}$ (Fig. 3 ) in the measurement of x-rays of a few keV [3]. This resolution is already 20 times greater than for any existing semiconductor detector.

More interesting for underground cryogenics is the successful construction of large detectors using materials with both low and high atomic numbers $Z$ [6], where masses up to about 340 grams have been reached [7].

\section{Applications}

\section{Dark matter searches}

It is well known that the density in the Universe of the visible (more correctly, the baryonic) matter is at least one order of magnitude less than the critical value needed to "close" the expansion of the Universe. More significant, at least for an experimentalist, is the fact that various measurements such as those on the rotation of galaxies and on the relative motion of single galaxies of the same group, indicate that the average density of matter in the Universe is an order of magnitude larger than the baryonic density. The origin of this extra mass and the nature of dark matter are still unclear. Candidates for dark matter include massive, but light, neutrinos, heavy neutrinos, small black holes, and new but still undiscovered particles such as those predicted by theories for supersymmetry that try to unify all the forces in Nature. The solar system and Earth presumably move with respect to these particles, which should then be revealed by their interactions in a suitable detector placed underground and shielded against radioactivity [4]. If dark matter consists of WIMPS (Weak Interacting Massive Particles) the only way of detecting them directly is via their elastic scattering on a nucleus in a detector where the nuclear recoil energy is measured. Some experiments have already been carried out by searching for these recoils in semiconductor

Fig. 4-Cryogenic $\mathrm{TeO}_{2}$ detectors that have been developed by the Milan group to search for double-beta decay (a, upper) and to perform gamma-ray spectroscopy (b, lower). The diagram gives a cross-section through the copper frame holding the $\mathrm{TeO}_{2}$ crystal which was used for a); the photograph shows a similar detector that was employed for $b$ ). detectors placed underground. Results are still negative. We have already established, however, that thermal detectors are likely to be more effective than the classical ones for revealing nuclear recoils, and therefore dark matter [8].

\section{Searching for rare events}

Let us take double-beta decay (DBD) as an example of a rare event. This process is very important in the framework of the conservation of the lepton number, where two electrons are emitted simultaneously. Neutrinoless DBD, if observed, would signal the violation of lepton number conservation and the existence of massive Majorana neutrinos - unambiguous evidence of a new physics beyond the Standard Model [9]. There has been a suggestion to use for this process a detector made with a DBD active material that would act at the same time as source of the decay and as a detector to reveal it. Many materials are good candidates for DBD and possess at the same time mechanical and thermal properties that make them suitable for constructing highperformance croygenic bolometers. In particular, the group from Milan recently installed in the Gran Sasso Laboratory, Assergi, Italy, a DBD experiment based on ${ }^{130} \mathrm{Te}$ in the form of a $20.9 \mathrm{~g} \mathrm{TeO}_{2}$ crystal mounted in a dilution refrigerator (Fig. $4 \mathrm{a}$ ). It has achieved a sensitivity three orders of magnitude better than was reported previously [10].

\section{High-energy qamma-ray detection}

It is well known that gamma rays are normally detected by searching for peaks in energy due to interactions in a detector involving the photoelectric effect and pair formation. The cross-sections for these processes increase strongly with atomic number. Excellent gamma-ray spectroscopy can therefore be carried out with thermal bolometers constructed using a high- $Z$ material, as has already been demonstrated for a tellurium oxide detector (Fig. 4b) made by the Milan group [11].
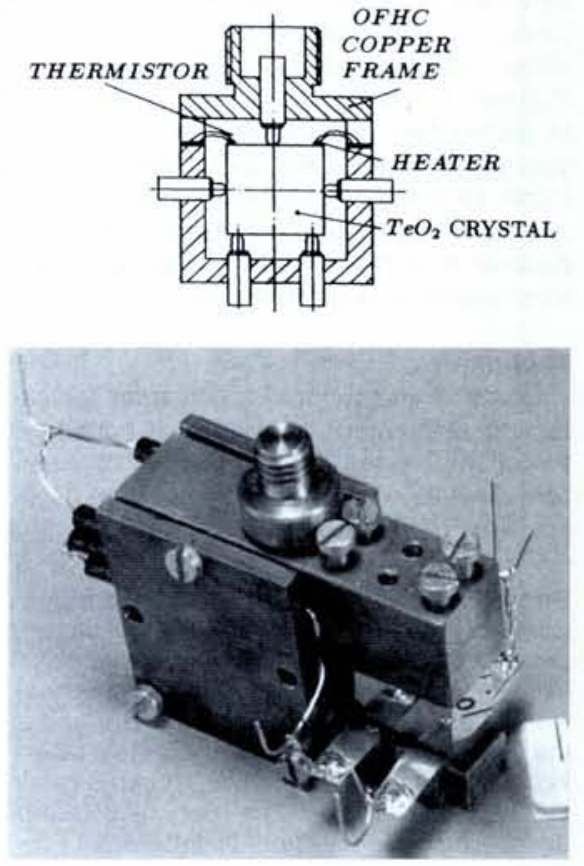


\section{Underground Cryogenics}

As the events being sought in experiments on dark matter or double-beta decay are rare, and since the pulses are long, it is essential to reduce spurious counting arising from cosmic rays and environmental radioactivity. The dilution refrigerator being operated at Gran Sasso by the Milan group is in fact the world's first deep underground cryogenic plant (Fig. 5). The device is constructed from specially selected materials having low levels of radioactivity. A high- $Z$ bolometer with a $\mathrm{TeO}_{2}$ detector crystal weighing $340 \mathrm{~g}$ has been installed. This may represent the beginning of "underground cryogenics", the importance of which could extend well beyond the field of nuclear and subnuclear physics.

Consider, as a final example, attempts to reach very low temperatures (tens of microkelvin) where up to now heat from radioactivity and cosmic rays has been neglected. In a normal laboratory at the Earth's

Fig. 5 - Schematic illustration of the Milan group's underground detector operating at Gran Sasso laboratory. The large $(340 \mathrm{~g})$ tellurium oxide crystal is supported inside a specially constructed, shielded dilution refrigerator. surface without special shields, the thermal energies delivered by cosmic rays and by radioactivity are comparable, amounting in total to $0.3 \mathrm{pW} / \mathrm{g}$ : this is only an order of magnitude less than the total heat leak encountered experimentally in the microkelvin region. It will therefore become essential in the near future to carry out searches for rare events in an underground laboratory where contributions from cosmic rays and radioactivity are reduced by many orders of magnitude. This single example shows how the joint effort of cryogenics, particle physics and astrophysics should lead to exciting and perhaps unexpected results.

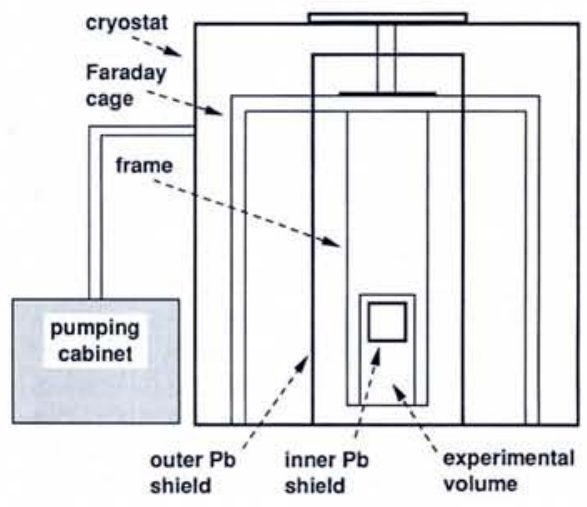

[1] Fiorini E. and Niinikoski T.O., Nucl. Intr. \& Methods 224 (1984) 83.

[2] Moseley S.M., Mather J.C. and McCammon D., J. Appl. Phys. 56 (1984) 1257.

[3] Proc. 3rd Int. Workshop on Low Temperature Detectors for Neutrinos and Dark Matter, Eds: L. Brogiato, D.V. Camin and E. Fiorini (Editions Frontières) 1990.

[4] Fiorini E., Proc. 2nd Conf. of the European Materials Res. Soc., Strasbourg, 1988 (Elsevier,1989).

[5] Camin D.V., Pessina G. and Previtali E., Nucl. Instr. \& Methods A 315 (1992) 385.

[6] Fiorini E., Physica B 167 (1991) 388; Proc. Low Temperature Detectors for Neutrinos \& Dark Matter IV, Eds.: N.E. Both \& G. Salmon (Editions Frontières) 1992.

[7] Alessandrello A., et al., in Proc. 24th Int. Conf. on High Energy Physics (Dallas, USA, 1992), to be published.

[8] Smith P.F. and Levin J.D., Phys. Rep. 187 (1990) 283; Rich J., Relativistic Astrophysics, Cosmlogy and Fundamental Physics, Ann. New York Acad. of Sciences (New York, NY, USA) 1991, p. 357.; Fiorini E., ibid., p. 446. [9] Tomoda T., Rep. Prog. Phys. 54 (1991) 53; Moe M.K., Nuclear Phys. B (Proc. Suppl.) 19 (1991) 158; Fiorini E., Nucl. Phys. News 1 (1991) 17; Klapdor H.V., J. Phys. G: Part. Phys. 17 (1991) 1.

[10] Alessandrello A., et al., Phys. Lett. B 285 (1992) 176

[11] Alessandrello A., et al., Nucl. Instr. \& Methods A 320 (1992) 388.

\section{Cosmology with Supernovae}

Hans Hippelein from the Max Planck Institute for Astronomy in Heidelberg, Germany, describes the significance of a recent observation of a Type 1a supernova "candle" at a record distance.

Type 1a supernovae (SN1a) are the result of deflagration and detonation of accreting stars. These exploding stars shine half as brightly as a whole galaxy (maximum brightness $M_{\mathrm{B}}=19.8 \mathrm{mag}$ ) for about a week and can be easily detected up to a redshift of $z \approx$ 0.5 , corresponding to a distance of about $5.10^{9}$ light years. The scatter in maximum brightness is small $(0.5 \mathrm{mag} ; \approx 25 \%$ ) because all Type 1a supernovae arise from exactly the same kind of star: a white dwarf which is fed by material from a companion star until it reaches a critical mass of 1.4 times that of the sun and explodes.

Because of the high brightness and the small scatter in brightness, $\mathrm{SN} 1 \mathrm{a}$ are probably the best "candles" for studying the expansion of the universe. At distances $z=$ 0.5 , the cosmological effect of deceleration of the expansion of the universe due to gravitational forces between galaxies is comparable to the variation in the maximum brightness of supernovae.

By observing a large number of supernovae one can plot a curve describing the apparent brightness as a function of the redshift (see figure). The deceleration parameter $q_{0}$ is given by the slope of the curve, without knowing the Hubble constant which relates redshift and the distance of galaxies in the expanding universe. Using $q_{0}$, astronomers can directly estimate $\Omega$, the mean mass density parameter in the universe (e.g., for the Friedman model $\Omega=2 q_{0}$ ).

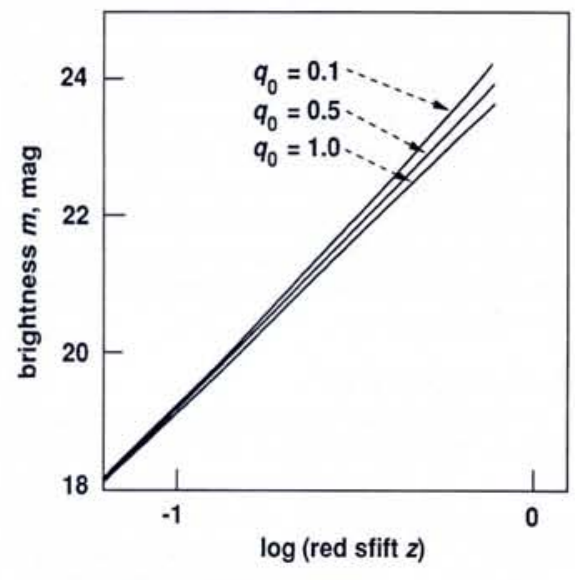

Apparent brightness $m$ of Type 1a supernovae versus redshift $z$ (logarithmic scale) for a Hubble constant of $50 \mathrm{~km} \mathrm{~s}^{-1} \mathrm{Mpc}^{-1}$ for different values of the deceleration parameter $q_{0}$ which is directly related to a parameter $\Omega$ describing the mean density of matter in the universe. By plotting $m$ for different Type 1a supernovae as a function of the observed $z$ one can therefore in principle determine $q_{0}$. and hence $\Omega$, without knowing the value of the Hubble constant.

For $\Omega<1$, the universe will expand forever and for $\Omega>1$ the expansion will eventually halt and reverse course into a contraction. The observation of luminous matter in the universe gives $\Omega=0.2$. Many astronomers, however, favour a higher density $(\Omega=1)$ because it is difficult to form galaxies with a smaller value. This higher value is only possible by introducing hypothetical "dark" matter which interacts with the visible matter through gravitational forces.

In April 1992, S. Pearlmutter, C. Pennypacker, G. Goldhaber, and other astronomers from Berkeley, USA, together with scientists from Cambridge and Durham in the UK and from Stockholm used the $2.5 \mathrm{~m}$ Isaac Newton Telescope in La Palma, Canary Islands, for one week to see if they could apply the new method. They scanned 10000 deep-space galaxies in a search for Type 1a supernovae, where the probability of locating a SN1a is only about one per galaxy in 500 years. Pearlmutter and colleagues were therefore extremely lucky when they had a detection, near the border between the Hercules and Corona Borealis constellations, with $z=0.457$ determined from the Doppler broadening of spectral lines. The measured $z$ is considerably larger than the previous record of 0.31 for Type 1 supernovae. However, this single observation is insufficient for discriminating between density parameters of 0.2 and 1 .

Pearlmutter says that the detection of 25 Type 1a supernovae (which requires about one to two years of observation) would allow $\Omega$ to be pinned down to within about $30 \%$, sufficient to distinguish between a low-density universe with $\Omega \approx 0.2$ and a higher density one with $\Omega=1$. The number 25 seems optimistic considering absorption and other effects that have to be allowed for. In principle, however, the method should work and be able to solve one of today's major problems in astronomy within a few years. 